Draft VERSion JANUARY 3, 2022

Typeset using $\mathrm{LAT}_{\mathrm{E}} \mathrm{X}$ preprint2 style in AASTeX63

\title{
Identification of active magnetic reconnection using magnetic flux transport in plasma turbulence
}

\author{
TAK Chu Li, ${ }^{1}$ Yi-Hsin LiU, ${ }^{1}$ AND Yi QI ${ }^{2}$ \\ ${ }^{1}$ Department of Physics and Astronomy, Dartmouth College, Hanover, NH, USA \\ ${ }^{2}$ Department of Earth, Planetary, and Space Sciences, University of California, Los Angeles, CA, USA
}

\begin{abstract}
Magnetic reconnection has been suggested to play an important role in the dynamics and energetics of plasma turbulence by spacecraft observations, simulations and theory over the past two decades, and recently, by magnetosheath observations of MMS. A new method based on magnetic flux transport (MFT) has been developed to identify reconnection activity in turbulent plasmas. This method is applied to a gyrokinetic simulation of two-dimensional (2D) plasma turbulence. Results on the identification of three active reconnection X-points are reported. The first two X-points have developed bi-directional electron outflow jets. Beyond the category of electron-only reconnection, the third $\mathrm{X}$-point does not have bi-directional electron outflow jets because the flow is modified by turbulence. In all cases, this method successfully identifies active reconnection through clear inward and outward flux transport around the X-points. This transport pattern defines reconnection and produces a new quadrupolar structure in the divergence of MFT. This method is expected to be applicable to spacecraft missions such as MMS, Parker Solar Probe, and Solar Orbiter.
\end{abstract}

Keywords: magnetic reconnection — turbulence — plasmas

\section{INTRODUCTION}

Magnetic reconnection and plasma turbulence are both fundamental processes ubiquitously operating throughout the universe. Reconnection has been suggested to contribute to energy dissipation (Dmitruk et al. 2004; Sundkvist et al. 2007; Osman et al. 2011, 2012; Markovskii \& Vasquez 2011; Perri et al. 2012; Wan et al. 2012; Karimabadi et al. 2013; TenBarge \& Howes 2013; Wu et al. 2013; Zhdankin et al. 2013; Shay et al. 2018) and potential changes in the cascade (Loureiro \& Boldyrev 2017; Boldyrev \& Loureiro 2017; Mallet et al. 2017a; Franci et al. 2017; Mallet et al. 2017b; Loureiro \& Boldyrev 2017; Vech et al. 2018; Stawarz et al. 2019) of turbulence by in situ observations, numerical simulations and theory. In heliospheric turbulence, reconnection was first observed in situ in the terrestrial magnetosheath by Cluster (Retinò et al. 2007). Recently, high resolution measurements from MMS (Burch et al. 2016) have enabled the detection of electron jets in small-scale current sheets in the turbulent magnetosheath (Yordanova et al. 2016; Vörös et al. 2017; Phan et al. 2018;

Corresponding author: Tak Chu Li

tak.chu.li@dartmouth.edu
Wilder et al. 2018), including notably, electron-only reconnection (Phan et al. 2018).

Reconnection occurs in a small-scale electron diffusion region (EDR) within a thin current sheet. As upstream field lines flow into the EDR, they reconnect at the Xpoint. The reconnected field possesses strong magnetic tension, which drives the reconnected field away from the X-point, ejecting plasma that is coupled to it as bi-directional outflow jets. The fundamental process of reconnection can be described as inward and outward transport of magnetic flux and associated plasmas at an $\mathrm{X}$-point. The transport of magnetic flux and plasma flows across a separatrix was used to to define reconnection (Vasyliunas 1975).

At the frontier of turbulence and reconnection research, important questions include how reconnection occurs in a dynamical turbulent system and how the rich dynamics of turbulence and reconnection, such as turbulent energy dissipation and cascade, interplay. Nevertheless, there is still no clear, reliable method to identify reconnection X-points in turbulent plasmas. In $2 \mathrm{D}$ turbulence simulations, the method of saddle points that define an X-point topology was applied (Servidio et al. 2009; Servidio et al. 2010; Wan et al. 2013; Haggerty et al. 2017). However, among a large number of identified X-points, only a few displayed significant recon- 
nection electric fields (Servidio et al. 2009). It would be possible that many identified X-points are not actively reconnecting.

In observations, a commonly used method to identify reconnection is the detection of bi-directional Alfvénic ion outflow jets. In a turbulent system such as the terrestrial magnetosheath, reconnection can happen at subion or electron scales (Wilder et al. 2018; Phan et al. 2018), and electron jets becomes the conclusive signature of reconnection. However, fast turbulent flows at sub-ion scales can make the detection challenging. In fact, only one out of several tens of sub-ion-scale current sheets detected by Phan et al. (2018) displayed clear bidirectional reconnection electron jets.

Recently, the transport of magnetic flux around an $\mathrm{X}$-point was considered in kinetic simulations of reconnection (Liu \& Hesse 2016; Liu et al. 2018). MFT takes into account the decoupling of electron flow and magnetic flux (slippage) arising from a non-ideal electric field, and thus correctly captures the inward and outward transport of magnetic flux around a reconnection $\mathrm{X}$-point. In a symmetric reconnection simulation with shear flows, the electron flow can be highly distorted (Liu et al. 2018). Under stronger shear flows or asymmetry likely in turbulence, the electron flow may not show typical reconnection outflows. In fact, in a highly asymmetric configuration, active reconnection with only one electron jet is possible (Liu \& Hesse 2016).

\section{THEORY}

The transport of magnetic flux inherent to reconnection represents an innovative way for identifying active reconnecting $\mathrm{X}$-points in turbulence. The presence of inward flux transport also indicates reconnection is actively taking place. The MFT velocity $\mathbf{U}_{\psi}$ was previously derived in one and two dimensions (Liu \& Hesse 2016; Liu et al. 2018). The key steps leading to the definition of $\mathbf{U}_{\psi}$ are summarized here. In $2 \mathrm{D}$, the magnetic field can be represented as an inplane and out-of-plane (guide field) component directed along $\hat{\mathbf{z}}: \quad \mathbf{B}=\hat{\mathbf{z}} \times \nabla \psi+B_{0} \hat{\mathbf{z}}$. Curling the Faraday's law: $\hat{\mathbf{z}} \times\left[\partial_{t} \mathbf{B}+c \nabla \times \mathbf{E}=0\right]$ results in $\partial_{t} \psi=c E_{z}$. We then consider the electron momentum equation: $\mathbf{E}+\mathbf{v}_{e} \times \mathbf{B} / c=\mathbf{E}_{e}^{\prime}$, where $\mathbf{E}_{e}^{\prime}$ is the non-ideal electric field in the electron frame. Taking the $z$ component of this equation and casting it into the form of the $2 \mathrm{D}$ advection equation of magnetic flux: $\partial_{t} \psi+\mathbf{U}_{\psi} \cdot \nabla_{\perp} \psi=0$, the in-plane MFT velocity is then given by:

$$
\mathbf{U}_{\psi} \equiv \mathbf{v}_{e p}-\left(\mathbf{v}_{e p} \cdot \hat{b}_{p}\right) \hat{b}_{p}+\frac{c E_{e z}^{\prime}}{B_{p}}\left(\hat{\mathbf{z}} \times \hat{b}_{p}\right),
$$

where $\hat{b}_{p} \equiv \mathbf{B}_{p} / B_{p}$ is the unit vector of the in-plane magnetic field $\mathbf{B}_{p}$ and $\mathbf{v}_{e p}$ the in-plane electron flow. The first two terms represent the in-plane electron flow perpendicular to $\mathbf{B}_{p}$. They come from the $\mathbf{v}_{\mathbf{e}} \times \mathbf{B}$ term in the electron momentum equation. For $\mathbf{E}_{e}^{\prime}=0$, the electron flow is frozen-in to the magnetic field and they move together. When $\mathbf{E}_{e}^{\prime} \neq 0$, slippage between magnetic flux and electron flow arises as the last term. Without separating the perpendicular electron flow and slippage terms, which provide a relation between the transport of magnetic flux and electron flow, Equation (1) can be simplified to:

$$
\mathbf{U}_{\psi}=\frac{c E_{z}}{B_{p}}\left(\hat{\mathbf{z}} \times \hat{b}_{p}\right) .
$$

To the first order in gyrokinetics, $\mathbf{U}_{\psi}$ is given by Equation (1) or (2) with $\mathbf{v}_{e p}, \mathbf{B}_{p}$ and $E_{e z}^{\prime}$ replaced by $\delta \mathbf{u}_{e p}$, $\delta \mathbf{B}_{p}$ and $\delta E_{e z}^{\prime}=\delta E_{z}+\left(\delta \mathbf{u}_{e p} \times \delta \mathbf{B}_{p} / c\right)_{z}$, where fluctuating quantities in turbulence are the in-plane electron bulk flow $\delta \mathbf{u}_{e p}$ and so on. Note that Equation (1) is not applicable at the X-point because a source or sink term, representing flux generation or annihilation at the $\mathrm{X}$-point, is not included in this advection equation.

A new quantity, the divergence of MFT, $\nabla \cdot \mathbf{U}_{\psi}$, is considered here. $\nabla \cdot \mathbf{U}_{\psi}<0$ and $>0$ can capture the converging inflows and diverging outflows of magnetic flux, respectively. These bi-directional inflows and outflows of magnetic flux at an X-point signifies active reconnection. $\nabla \cdot \mathbf{U}_{\psi}$ also informs about the time scale of diverging magnetic flux from the X-point. Having the dimension of inverse time, $\nabla \cdot \mathbf{U}_{\psi}$ is frame-independent in $2 \mathrm{D}$ in the non-relativistic limit. Therefore, one can compute $\nabla \cdot \mathbf{U}_{\psi}$ for moving X-points without changing frames.

\section{CODE}

The 2D gyrokinetic turbulence simulation has been previously performed ( $\mathrm{Li}$ et al. 2016) using the the Astrophysical Gyrokinetics Code, or AstroGK, described in details in (Numata et al. 2010). AstroGK has been extensively used to investigate turbulence in weakly collisional plasmas (Howes et al. 2008; Tatsuno et al. 2009; Howes et al. 2011; TenBarge \& Howes 2012; Nielson et al. 2013; TenBarge \& Howes 2013; Howes 2016; Li et al. 2016; Howes et al. 2018; Li et al. 2019) and collisionless strong-guide-field reconnection (Numata et al. 2011; TenBarge et al. 2014; Kobayashi et al. 2014; Numata \& Loureiro 2015). AstroGK is an Eulerian continuum code with triply periodic boundary conditions. It has a slab geometry elongated along the straight, uniform background magnetic field, $\mathbf{B}_{0}=B_{0} \hat{\mathbf{z}}$. The code evolves the perturbed gyroaveraged Vlasov-Maxwell equations in five-dimensional phase space (three-dimensional-twovelocity) (Frieman \& Chen 1982; Howes et al. 2006). 
The evolved quantities are the electromagnetic gyroaveraged complementary distribution function for each species $s$, the scalar potential $\varphi$, parallel vector potential $A_{\|}$and parallel magnetic field perturbation $\delta B_{\|}$, where $\|$ is along the total local magnetic field $\mathbf{B}=B_{0} \hat{\mathbf{z}}+\delta \mathbf{B}$. The total and background magnetic fields are the same to first-order accuracy retained for perturbed fields in gyrokinetics. The velocity grid is specified by pitch angle $\lambda=v_{\perp}^{2} / v^{2}$ and energy $\varepsilon=v^{2} / 2$. The background distribution functions for both species are stationary uniform Maxwellians. Collisions are incorporated using a fully conservative, linearized gyro-averaged Landau collision operator (Abel et al. 2008; Barnes et al. 2009).

\section{SETUP}

The 2D Orszag-Tang Vortex (OTV) problem has been widely used to study plasma turbulence (Politano et al. 1989; Dahlburg \& Picone 1989; Picone \& Dahlburg 1991; Politano et al. 1995; Grauer \& Marliani 2000; Mininni et al. 2006; Parashar et al. 2009, 2014). It is given by

$$
\begin{aligned}
\delta \mathbf{u} & =\delta u\left[-\sin \left(k_{\perp} y\right) \hat{\mathbf{x}}+\sin \left(k_{\perp} x\right) \hat{\mathbf{y}}\right] \\
\delta \mathbf{B} & =\delta B\left[-\sin \left(k_{\perp} y\right) \hat{\mathbf{x}}+\sin \left(2 k_{\perp} x\right) \hat{\mathbf{y}}\right],
\end{aligned}
$$

where $\delta u=\delta B / \sqrt{4 \pi \rho_{0}}, \delta \mathbf{u}$ and $\delta \mathbf{B}$ are perturbations in the ion and electron bulk flow and the magnetic field, and $k_{\perp}=2 \pi / L_{\perp}$ are positive constants.

To follow the turbulent cascade from the inertial range $\left(k_{\perp} \rho_{i} \ll 1\right)$ to below electron scales $\left(k_{\perp} \rho_{e}>1\right)$ (TenBarge \& Howes 2013; TenBarge et al. 2013; TenBarge et al. 2014), we specify a reduced mass ratio, $m_{i} / m_{e}=$ 25 , which, in a simulation domain of $L_{\perp}=8 \pi \rho_{i}$ and dimensions $\left(n_{x}, n_{y}, n_{z}, n_{\lambda}, n_{\varepsilon}, n_{s}\right)=(128,128,2,64,32,2)$, enables us to resolve a dynamic range of $0.25 \leq k_{\perp} \rho_{i} \leq$ 10.5 , or $0.05 \leq k_{\perp} \rho_{e} \leq 2.1$. Plasma parameters are ion plasma $\beta_{i}=8 \pi n_{i} T_{0 i} / B_{0}^{2}=0.01$ and $T_{0 i} / T_{0 e}=1$. Collision frequencies of $\nu_{i}=10^{-5} \omega_{A 0}$ and $\nu_{e}=0.05$ $\omega_{A 0}$ (where $\omega_{A 0} \equiv k_{\|} v_{A}$ is a characteristic Alfvén wave frequency in $3 \mathrm{D}$ ) are sufficient to keep velocity space well resolved (Howes et al. 2008; Howes et al. 2011). Length, time and velocity are normalized to the ion gyroradius $\rho_{i} \equiv v_{t i} / \Omega_{c i}$, where $\Omega_{c i} \equiv e B_{0} / m_{i} c$, domain turnaround time $\tau_{0} \equiv L_{\perp} / \delta u$ and electron thermal speed $v_{t e} \equiv \sqrt{2 T_{0 e} / m_{e}} . \tau_{0}$ can be converted to the inverse ion gyro-frequency, a relevant time scale for reconnection, by $\tau_{0}=25 \Omega_{c i}^{-1}$. The divergence of velocity is normalized to $v_{t e} / \rho_{e}=\Omega_{c e}$.

\section{RESULTS}

Figure 1(a) shows the out-of-plane current density $J_{z}$ (color) and contours of the parallel vector potential $A_{\|}$ representing magnetic field lines of the OTV at an early

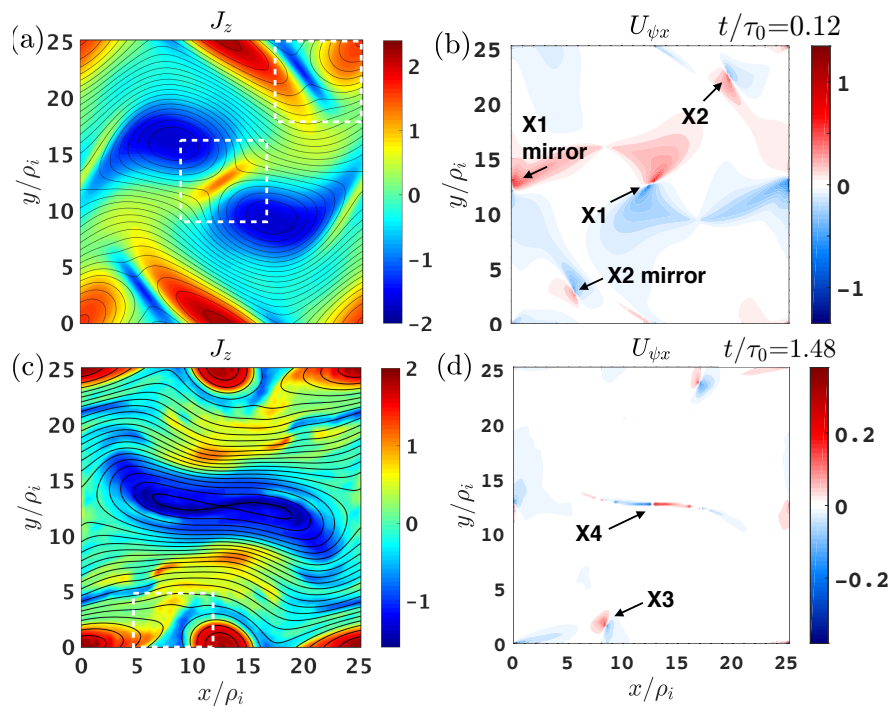

Figure 1. (a) The out-of-plane current density $J_{z}$ (color) overlaid with contours of $A_{\|}$of the OTV configuration, and (b) the $x$-component of $\mathbf{U}_{\psi}$ at $t / \tau_{0}=0.12$, showing $\mathrm{X} 1$ and $\mathrm{X} 2$ and their mirrors (labeled). At $t / \tau_{0}=1.48$, (c) $J_{z}$ showing developed turbulence and (d) $U_{\psi x}$ revealing X3, X4 (labeled) and X3 mirror. The bi-directional outflows of magnetic flux at $\mathrm{X} 1$ and $\mathrm{X} 4$, and inflows at $\mathrm{X} 2$ and $\mathrm{X} 3$ are observed. $\delta B_{p}$ is offsetted by adding a $1 \%$ of its maximum value in the domain such that $\mathbf{U}_{\psi}$ remains finite at the $\mathrm{X}$ - and O-points that have vanishing $\delta B_{p}$. This does not qualitatively affect the profile of $\mathbf{U}_{\psi}$. Dashed boxes indicate regions in zoomedin figures. See an animation of $J_{z}$ online. The animation lasts for $t / \tau_{0}=0-2.01$. It shows the evolution of reconnection driven by turbulent flows.

time of $t / \tau_{0}=0.12$. The OTV has an initial flow configuration that rotates the two vortices near the center of the domain, forming a current sheet in between. The symmetry of the two vortices allows symmetric reconnection to take place at the current sheet. The flows also drive two asymmetric vortices at the top right and bottom left, resulting in two mirroring asymmetric reconnection $\mathrm{X}$-points by symmetry of the system. A fourth reconnection $\mathrm{X}$-point, which is a mirror of the central symmetric reconnection $\mathrm{X}$-point, is located at $(x, y) \simeq(0,12.6)$. The central symmetric (X1) and top-right asymmetric (X2) $\mathrm{X}$-points are two of the cases we will discuss in details.

As the total turbulence energy dissipates over time ( $\mathrm{Li}$ et al. 2016), the driving of reconnection weakens and reconnection at later times is generally weaker than earlytime events. Figure $1(\mathrm{c})$ shows $J_{z}$ at late time $t / \tau_{0}=1.48$ when multiscale features, including small-scale current sheets, have developed. A turbulent cascade in the dissipation range (see Figure A1 for the magnetic energy spectrum) is also developed. At this time, an asymmetric reconnection $\mathrm{X}$-point forms at the bottom left. This $\mathrm{X}$-point (X3) does not develop bi-directional electron 

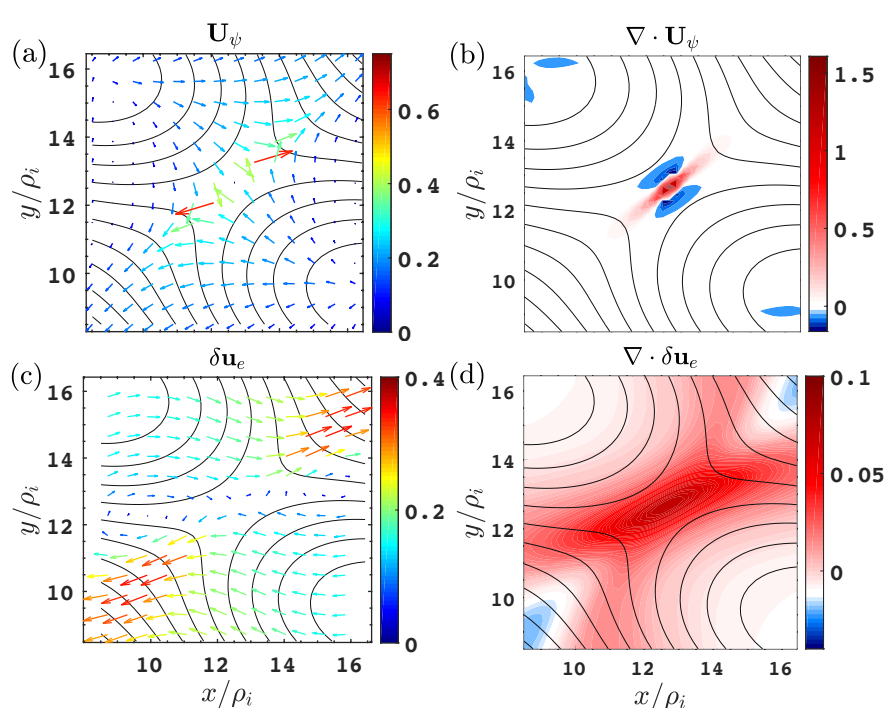

Figure 2. Application of the MFT method to X1, a symmetric reconnection $\mathrm{X}$-point. Plotted quantities are (a) vectors of $\mathbf{U}_{\psi}$, (b) the divergence of $\mathbf{U}_{\psi}$, (c) vectors of the fluctuating in-plane electron flow velocity $\delta \mathbf{u}_{e}$, and (d) the divergence of $\delta \mathbf{u}_{e}$, overlaid with $A_{\|}$contours. The amplitudes of vectors are denoted by the color and relative length of the arrows. The divergence of velocity is normalized to $v_{t e} / \rho_{e}$.

outflow jets and therefore cannot be identified through electron flows. Below we discuss the application of MFT and the identification of each reconnection X-point.

Figure 1(b) shows the $x$-component of the MFT velocity, $U_{\psi x}$, of the whole domain at $t / \tau_{0}=0.12$, showing $\mathrm{X} 1$ and $\mathrm{X} 2$ as well as their mirrors, and (d) at $t / \tau_{0}=1.48$, showing X3, its mirror and a reconnection X-point (X4) formed at the center of an evolved, elongated vortex (flux tube). The factor of $\delta B_{p}^{-1}$ in the definition of $\mathbf{U}_{\psi}$ could tend to infinity at the X-and O-points where $\delta B_{p}$ vanishes. As a practical step, we add a $1 \%$ offset to $\delta B_{p}$ everywhere so that $\mathbf{U}_{\psi}$ remains finite at the $\mathrm{X}$ and $\mathrm{O}$-points that have vanishing $\delta B_{p}$. For the range of $0.01-4 \%$ offsets, the amplitudes of $\mathbf{U}_{\psi}$ and $\nabla \cdot \mathbf{U}_{\psi}$ only vary by a factor of 2 . Note that masking the $\mathrm{X}$-points by a grid point of size $\rho_{e}$ yields similar amplitudes to applying a $1 \% \delta B_{p}$ offset. Below we zoom in to X1-X3 to investigate the $\mathrm{X}$-points more thoroughly.

\subsection{X1: Symmetric reconnection X-point}

Figure 2 shows (a) vectors of $\mathbf{U}_{\psi}$, (b) $\nabla \cdot \mathbf{U}_{\psi}$, and for comparison, (c) vectors and (d) the divergence of $\delta \mathbf{u}_{\mathbf{e}}$ in a zoomed-in region around X1. Clear bi-directional outflows and converging inflows of magnetic flux around $\mathrm{X} 1$ are captured in $\mathbf{U}_{\psi}$. (b) $\nabla \cdot \mathbf{U}_{\psi}$ reveals negative (blue) and positive (red) amplitudes highly localized to $\mathrm{X} 1$, representing converging inward and diverging outward MFT at the X-point. This is the inherent flux transport pattern of reconnection. It results in a new
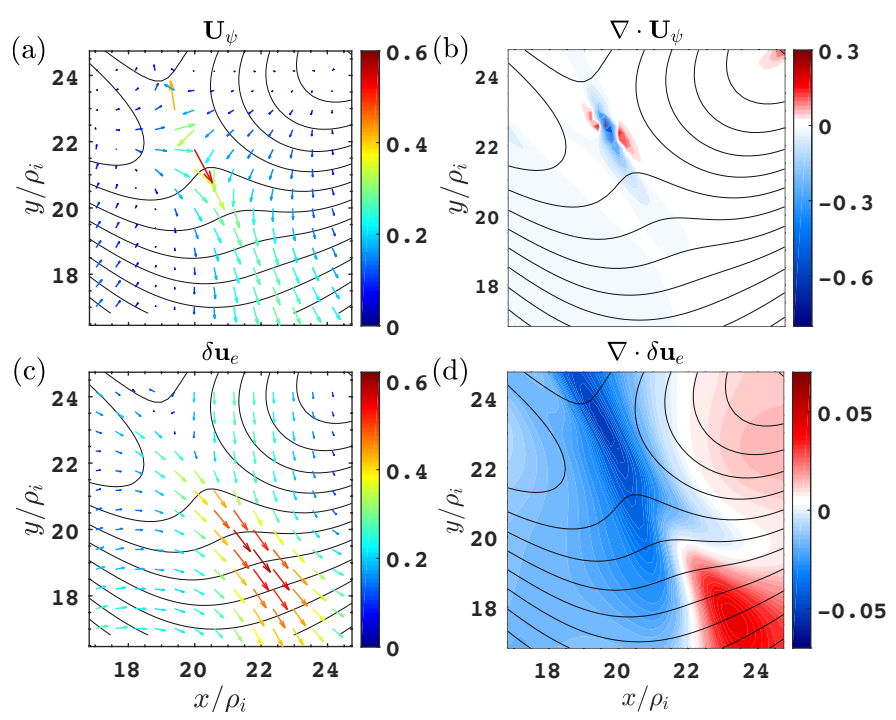

Figure 3. Same quantities as Figure 2 plotted for $\mathrm{X} 2$, an asymmetric reconnection X-point.

quadrupolar structure in $\nabla \cdot \mathbf{U}_{\psi}$. The quadrupolar structure reflects the bi-directional flux transport at the two sides upstream and downstream of the X-point. Both quantities are highly localized to the $\mathrm{X}$-point, and can serve as local signatures of reconnection.

Bi-directional electron outflow jets in the outflow region can be seen in (c) $\delta \mathbf{u}_{\mathbf{e}}$. (d) $\nabla \cdot \delta \mathbf{u}_{e}$ reveals positive amplitude, representing the diverging outflows. In comparison to $\mathbf{U}_{\psi}$, the electron outflow develops further from the $\mathrm{X}$-point and over a much broader region.

\subsection{X2: Asymmetric reconnection X-point}

The same quantities as Figure 2 are plotted around $\mathrm{X} 2$ in Figure 3. Similarly, clear bi-directional inflows and asymmetric bi-directional outflows of magnetic flux are captured in (a) $\mathbf{U}_{\psi}$, with the downward transport being stronger. (b) $\nabla \cdot \mathbf{U}_{\psi}$ reveals the presence of converging inward and diverging outward flux transport as $\nabla \cdot \mathbf{U}_{\psi}<0$ and $>0$, respectively, at X2. Both signify active reconnection.

In (c) $\delta \mathbf{u}_{\mathbf{e}}$, asymmetric electron outflow jets are seen, with a stronger downward jet from X2. (d) The divergence of the electron flow reveals negative and positive amplitudes located broadly around and downstream from the X-point, representing converging inflows and diverging outflows of electrons at this $\mathrm{X}$-point.

\subsection{X3: Reconnection X-point Without bi-directional plasma jets}

As the turbulent flows that drive reconnection are significantly dissipated at late times ( $\mathrm{Li}$ et al. 2016), reconnection activity becomes weaker than early-time reconnection. Nevertheless, converging inflows and bi- 

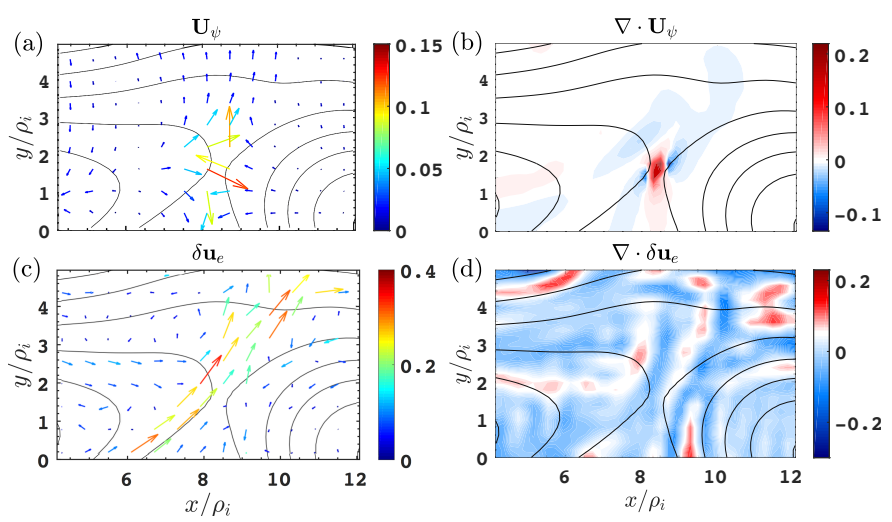

Figure 4. Same format as Figure 2 plotted for X3, a reconnection $\mathrm{X}$-point with only one outflow jet in electrons (and ions), at $t / \tau_{0}=1.48$. Velocity vectors are measured at the X-point frame.

directional outflows of magnetic flux are captured in Figure 4 (a) $\mathbf{U}_{\psi}$ at $\mathrm{X} 3$. (b) $\nabla \cdot \mathbf{U}_{\psi}$ also reveals positive and negative amplitudes highly localized to the X-point, sharing a similar quadrupolar structure to X2 and X1.

On the other hand, the electron flow is highly modified by turbulence. No clear bi-directional outflow jets are seen in the electrons (or in the ions (Appendix Figure A2)) at X3. Only one electron and one ion outflow jet are present. (d) $\nabla \cdot \delta \mathbf{u}_{e}$ also does not show clear evidence of reconnection. Plasma flows cannot be used for identifying reconnection at this X-point. However, the MFT method is able to identify reconnection through its clear inward and outward flux transport at this X-point, demonstrating the sensitivity of MFT in identifying reconnection activity in turbulence.

\subsection{Super-Alfvénic $\mathbf{U}_{\psi}$}

While $\mathbf{U}_{\psi}$ is normalized to $v_{t e}$, it is meaningful to compare it with the upstream Alfvén speed. Using the electron plasma $\beta_{e} \equiv\left(v_{t e} / c_{A e}\right)^{2}=0.01$, where $c_{A e}=$ $\sqrt{B_{0} / 4 \pi n_{0} m_{e}}$, in the simulation, and estimates of the upstream $\delta B_{p} / B_{0} \sim 0.1$ and density $n / n_{0} \sim 0.7-1.1$ for the three X-points, we can relate the upstream electron Alfvén speed (Cassak \& Shay 2007) to $v_{t e}$ as $c_{A e, p} / v_{t e} \sim$ 1. Therefore, at $\mathrm{X} 1$ and $\mathrm{X} 2, U_{\psi}$ is of order $c_{A e, p}$. The flux transport velocity is electron Alfvénic. Similarly, at $\mathrm{X} 3, U_{\psi} \sim 1.2 c_{A, p}$ is super-Alfvénic. The higher velocity at early-time reconnection is associated with strong driving by initial turbulent flows. The Alfvénic velocity at late times is consistent with undriven reconnection simulations (Liu \& Hesse 2016). $\mathbf{U}_{\psi}$ is between orders $c_{A, p}$ and $c_{A e, p}$ based on the simulation.

\subsection{Divergence of MFT}

Plotted in Figure 5 is the divergence of MFT of the whole domain at (a) $t / \tau_{0}=0.12$, showing $\mathrm{X} 1$ and $\mathrm{X} 2$
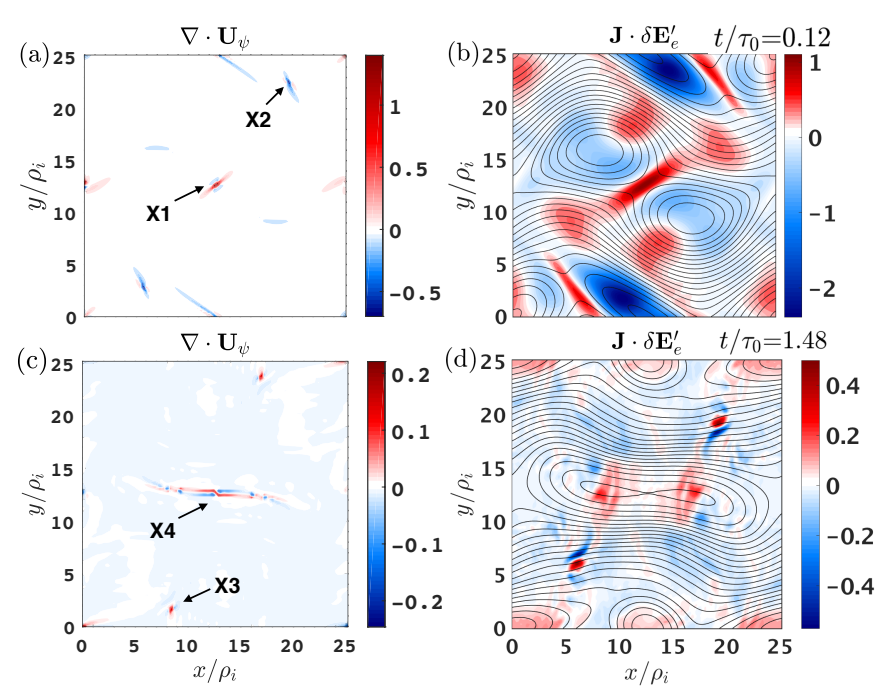

Figure 5. (a) The divergence of MFT of the whole domain at (a) $t / \tau_{0}=0.12$, showing X1 and X2 (labeled) and their mirrors, and (c) $t / \tau_{0}=1.48$, showing X3 and X4 (labeled) among the turbulence; (b) and (d) $\mathbf{J} \cdot \delta \mathbf{E}_{e}^{\prime}$, the non-ideal energy conversion in the electron frame, at the two times.

and their mirrors, and at (c) $t / \tau_{0}=1.48$, when turbulence is developed, revealing X3 and X4. $\nabla \cdot \mathbf{U}_{\psi}$ shows significant amplitudes only at the active reconnection $\mathrm{X}$-points, even among the turbulence. It remains small throughout the domain, and is thus suitable for the identification of reconnecting X-points in turbulence. $\nabla \cdot \delta \mathbf{u}_{e}$ is much more structured throughout the system, and at late times, becomes highly turbulent (not shown). For comparison, (b) $\mathbf{J} \cdot \delta \mathbf{E}_{e}^{\prime}$, energy conversion (Zenitani et al. 2011) in the electron frame, is much more broadly distributed over the current sheets and throughout the system. (d) At late times, it is dominated by turbulent flows far away from the reconnection X-points, and thus may not help in locating reconnection in turbulence. The amplitude of $\nabla \cdot \mathbf{U}_{\psi}$ is of order $0.1-1 \Omega_{c e}$ at the three reconnection X-points.

\section{DISCUSSION}

The flux transport velocity has been generally considered as the $\mathrm{E} \times \mathrm{B}$ drift velocity. In Equation (1), the slippage between magnetic flux and electron flow arising from an non-ideal electric field $\mathbf{E}_{e}^{\prime}$ is included. For the three reconnection $\mathrm{X}$-points, the slippage provides the major contribution to the inflows and outflows of magnetic flux near the $\mathrm{X}$-point, being $\sim 2-3$ times larger than the perpendicular electron flow. Further away from the $\mathrm{X}$-point where the the slippage becomes small, $\mathbf{U}_{\psi}$ follows the perpendicular electron flow, which is mainly the $\mathrm{E} \times \mathrm{B}$ drift.

$\nabla \cdot \mathbf{U}_{\psi}$ consistently shows a quadrupolar structure at all reconnection X-points in turbulence. However, a sig- 
nal is possible at O-points, where magnetic flux annihilation could happen. This process is recently explored by MMS (Hasegawa et al. 2020). MFT activity at Opoints deserves future investigation.

A new category of reconnection in turbulence beyond electron-only reconnection (Phan et al. 2018) is revealed by X3. Only a single electron Alfvénic electron jet and Alfvénic ion jet are observed at X3. This category has $\mathbf{U}_{\psi}$ reversals, but no plasma outflow jet reversal. Electron-only reconnection with only one jet is also reported in simulations of shock-driven turbulence (Bessho et al. 2020).

\section{APPLICATION TO HELIOSPHERIC PLASMAS}

Application of the MFT method to heliospheric plasmas requires the following conditions: (i) $k_{\|} \ll k_{\perp}$, where "|" is along the background magnetic field, and (ii) the reconnection magnetic fields primarily reside on a local reconnection plane. $k_{\|} \ll k_{\perp}$ is based on $k_{\|} / k_{\perp} \ll \delta E_{\|} / \delta E_{\perp}$ for deriving $\partial_{t} \psi(\S 2)$, a condition well satisfied in the simulation. Equation (1) is then a good approximation of $\mathbf{U}_{\psi}$ even in $3 \mathrm{D}$ systems. Physically, this represents quasi-planar reconnection with parallel length scales much longer than perpendicular. $k_{\|} \ll k_{\perp}$ is well satisfied in the cascade of kinetic Alfvén wave turbulence (Cho \& Lazarian 2004; Schekochihin et al. 2009), which is consistent with solar wind and magnetosheath observations (Alexandrova et al. 2008; Alexandrova et al. 2009; Sahraoui et al. 2013; Chen 2016; Chen \& Boldyrev 2017). The model of planar reconnection is adopted by the local current sheet (LMN) coordinate (Sonnerup \& Cahill Jr. 1967), commonly used in space reconnection observations. Observations of re- connection in small-scale current sheets in the turbulent magnetosheath are consistent with this model (e.g. (Phan et al. 2018; Wilder et al. 2018)). Thus, the conditions for applying MFT is expected to be realistic for reconnection in heliospheric turbulence. Recent 3D PIC simulations further show that a long extended X-line, satisfying $k_{\|} \ll k_{\perp}$, easily arising in sub-ion-scale current sheets in 3D (Li et al. 2020), also favors reconnection activity (Liu et al. 2019; Huang et al. 2020).

\section{CONCLUSION}

The MFT method is a new way of identifying reconnection X-points in turbulent plasmas. It captures bi-directional inflows and outflows of magnetic flux at the X-points to signify reconnection, even without bidirectional plasma outflow jets. $\nabla \cdot \mathbf{U}_{\psi}$ is suitable for use in multi-spacecraft missions such as MMS. The first application to a $2 \mathrm{D}$ gyrokinetic turbulence simulation demonstrates the capability of this method in clearly capturing active reconnection signatures, as an inflowoutflow pattern or a quadrupolar structure in $\nabla \cdot \mathbf{U}_{\psi}$. It also reveals a new category of reconnection in turbulence beyond electron-only reconnection. This method has the potential to replace the plasma outflow jet reversal signature for reconnection. Applications to 3D simulations and heliospheric observations from spacecraft missions will present new opportunities to study the role of reconnection and identify new types of reconnection in turbulence.

The authors thank Tai Phan, Prayash Sharma Pyakurel and Daniel Verscharen for fruitful discussions. This work is supported by NSF award AGS-2000222 and NASA grants 80NSSC18K0754 and MMS mission 80NSSC18K0289.

\section{REFERENCES}

Abel, I. G., Barnes, M., Cowley, S. C., Dorland, W., \& Schekochihin, A. A. 2008, Phys. Plasmas, 15, 122509, doi: 10.1063/1.3046067

Alexandrova, O., Lacombe, C., \& Mangeney, A. 2008, Annales Geophysicae, 26, 3585,

doi: 10.5194/angeo-26-3585-2008

Alexandrova, O., Saur, J., Lacombe, C., et al. 2009,

Phys. Rev. Lett., 103, 165003, doi: 10.1103/PhysRevLett.103.165003

Barnes, M., Abel, I. G., Dorland, W., et al. 2009, Phys. Plasmas, 16, 072107, doi: 10.1063/1.3155085

Bessho, N., Chen, L. J., Wang, S., et al. 2020, Physics of Plasmas, 27, 092901, doi: 10.1063/5.0012443
Boldyrev, S., \& Loureiro, N. F. 2017, ApJ, 844, 125, doi: 10.3847/1538-4357/aa7d02

Burch, J. L., Tobert, R. B., Phan, T., Chen, L. J., \& Moore et al., T. E. 2016, Science, 10, 1126

Cassak, P. A., \& Shay, M. A. 2007, Phys. Plasmas, 14, 102114

Chen, C. H. K. 2016, Journal of Plasma Physics, 82, 535820602, doi: 10.1017/S0022377816001124

Chen, C. H. K., \& Boldyrev, S. 2017, The Astrophysical Journal, 842, 122, doi: 10.3847/1538-4357/aa74e0

Cho, J., \& Lazarian, A. 2004, Astrophys. J. Lett., 615, L41, doi: $10.1086 / 425215$

Dahlburg, R. B., \& Picone, J. M. 1989, Phys. Fluids B, 1, 2153, doi: 10.1063/1.859081 
Dmitruk, P., Matthaeus, W. H., \& Seenu, N. 2004, Astrophys. J., 617, 667, doi: 10.1086/425301

Franci, L., Cerri, S. S., Califano, F., et al. 2017, ApJL, 850, L16, doi: 10.3847/2041-8213/aa93fb

Frieman, E. A., \& Chen, L. 1982, Phys. Fluids, 25, 502

Grauer, R., \& Marliani, C. 2000, Phys. Rev. Lett., 84, 4850, doi: 10.1103/PhysRevLett.84.4850

Haggerty, C. C., Parashar, T. N., Matthaeus, W. H., et al. 2017, Physics of Plasmas, 24, 102308, doi: $10.1063 / 1.5001722$

Hasegawa, H., Denton, R. E., Genestreti, K. J., Nakamura, T. K. M., \& Phan et al., T. D. 2020, submitted to Nature

Howes, G. G. 2016, Astrophys. J. Lett., 82, L28, doi: 10.3847/2041-8205/827/2/L28

Howes, G. G., Cowley, S. C., Dorland, W., et al. 2006, Astrophys. J., 651, 590, doi: 10.1086/506172

Howes, G. G., Dorland, W., Cowley, S. C., et al. 2008, Phys. Rev. Lett., 100, 065004, doi: 10.1103/PhysRevLett.100.065004

Howes, G. G., McCubbin, A. J., \& Klein, K. G. 2018, Journal of Plasma Physics, 84, 905840105, doi: 10.1017/S0022377818000053

Howes, G. G., TenBarge, J. M., Dorland, W., et al. 2011, Phys. Rev. Lett., 107, 035004, doi: 10.1103/PhysRevLett.107.035004

Huang, K., Liu, Y.-H., Lu, Q., \& Hesse, M. 2020, Geophysical Research Letters, 47, e2020GL088147, doi: https://doi.org/10.1029/2020GL088147

Karimabadi, H., Roytershteyn, V., Wan, M., et al. 2013, Phys. Plasmas, 20, 012303, doi: 10.1063/1.4773205

Kobayashi, S., Rogers, B. N., \& Numata, R. 2014, Physics of Plasmas, 21, 040704, doi: 10.1063/1.4873703

Li, T. C., Howes, G. G., Klein, K. G., Liu, Y.-H., \& TenBarge, J. M. 2019, Journal of Plasma Physics, 85, 905850406, doi: 10.1017/S0022377819000515

Li, T. C., Howes, G. G., Klein, K. G., \& TenBarge, J. M. 2016, The Astrophysical Journal Letters, 832, L24. http://stacks.iop.org/2041-8205/832/i=2/a=L24

Li, T. C., Liu, Y.-H., Hesse, M., \& Zou, Y. 2020, J. Geophys. Res: Space Physics, 125, e2019JA027094, doi: 10.1029/2019JA027094

Liu, Y.-H., \& Hesse, M. 2016, Phys. Plasmas, 23, 060704

Liu, Y.-H., Hesse, M., Guo, F., Li, H., \& Nakamura, T. K. M. 2018, Physics of Plasmas, 25, 080701, doi: 10.1063/1.5042539

Liu, Y.-H., Li, T. C., Hesse, M., et al. 2019, J. Geophys. Res, 124, 2819, doi: 10.1029/2019JA026539

Loureiro, N. F., \& Boldyrev, S. 2017, Phys. Rev. Lett., 118, 245101, doi: 10.1103/PhysRevLett.118.245101
Loureiro, N. F., \& Boldyrev, S. 2017, ApJ, 850, 182, doi: 10.3847/1538-4357/aa9754

Mallet, A., Schekochihin, A. A., \& Chandran, B. D. G. 2017a, MNRAS, 468, 4862, doi: 10.1093/mnras/stx670

—. 2017b, Journal of Plasma Physics, 83, 905830609, doi: 10.1017/S0022377817000812

Markovskii, S. A., \& Vasquez, B. J. 2011, Astrophys. J., 739, 22, doi: 10.1088/0004-637X/739/1/22

Mininni, P. D., Pouquet, A. G., \& Montgomery, D. C. 2006, Phys. Rev. Lett., 97, 244503, doi: 10.1103/PhysRevLett.97.244503

Nielson, K. D., Howes, G. G., \& Dorland, W. 2013, Physics of Plasmas, 20, 072303, doi: 10.1063/1.4812807

Numata, R., Dorland, W., Howes, G. G., et al. 2011, Physics of Plasmas, 18, 112106, doi: 10.1063/1.3659035

Numata, R., Howes, G. G., Tatsuno, T., Barnes, M., \& Dorland, W. 2010, J. Comp. Phys., 229, 9347, doi: 10.1016/j.jcp.2010.09.006

Numata, R., \& Loureiro, N. F. 2015, Journal of Plasma Physics, 81, 3001, doi: 10.1017/S002237781400107X

Osman, K. T., Matthaeus, W. H., Greco, A., \& Servidio, S. 2011, Astrophys. J. Lett., 727, L11+, doi: 10.1088/2041-8205/727/1/L11

Osman, K. T., Matthaeus, W. H., Wan, M., \& Rappazzo, A. F. 2012, Phys. Rev. Lett., 108, 261102, doi: 10.1103/PhysRevLett.108.261102

Parashar, T. N., Shay, M. A., Cassak, P. A., \& Matthaeus, W. H. 2009, Phys. Plasmas, 16, 032310, doi: 10.1063/1.3094062

Parashar, T. N., Vasquez, B. J., \& Markovskii, S. A. 2014, Physics of Plasmas, 21, 022301, doi: 10.1063/1.4863422

Perri, S., Goldstein, M. L., Dorelli, J. C., \& Sahraoui, F. 2012, Phys. Rev. Lett., 109, 191101, doi: 10.1103/PhysRevLett.109.191101

Phan, T. D., Eastwood, J. P., Shay, M. A., Drake, J. F., \& Sonnerup et al., B. U. Ö. 2018, Nature, 557, 202, doi: 10.1038/s41586-018-0091-5

Picone, J. M., \& Dahlburg, R. B. 1991, Phys. Fluids B, 3, 29, doi: 10.1063/1.859953

Politano, H., Pouquet, A., \& Sulem, P. L. 1989, Phys. Fluids B, 1, 2330

—. 1995, Physics of Plasmas, 2, 2931, doi: 10.1063/1.871473

Retinò, A., Sundkvist, D., Vaivads, A., et al. 2007, Nature Physics, 3, 236, doi: 10.1038/nphys574

Sahraoui, F., Huang, S. Y., Belmont, G., et al. 2013, Astrophys. J., 777, 15, doi: 10.1088/0004-637X/777/1/15

Schekochihin, A. A., Cowley, S. C., Dorland, W., et al. 2009, Astrophys. J. Supp., 182, 310, doi: 10.1088/0067-0049/182/1/310 
Servidio, S., Matthaeus, W. H., Shay, M. A., Cassak, P. A., \& Dmitruk, P. 2009, Phys. Rev. Lett., 102, 115003

Servidio, S., Matthaeus, W. H., Shay, M. A., et al. 2010, Phys. Plasmas, 17, 032315, doi: 10.1063/1.3368798

Shay, M. A., Haggerty, C. C., Matthaeus, W. H., et al. 2018, Physics of Plasmas, 25, 012304, doi: 10.1063/1.4993423

Sonnerup, B. U. Ö., \& Cahill Jr., L. J. 1967, Journal of Geophysical Research (1896-1977), 72, 171, doi: 10.1029/JZ072i001p00171

Stawarz, J. E., Eastwood, J. P., Phan, T. D., Gingell, I. L., \& Shay et. al., M. A. 2019, ApJL, 877, L37, doi: $10.3847 / 2041-8213 / a b 21 c 8$

Sundkvist, D., Retinò, A., Vaivads, A., \& Bale, S. D. 2007, Phys. Rev. Lett., 99, 025004, doi: 10.1103/PhysRevLett.99.025004

Tatsuno, T., Dorland, W., Schekochihin, A. A., et al. 2009, PhRvL, 103, 015003, doi: 10.1103/PhysRevLett.103.015003

TenBarge, J. M., Daughton, W., Karimabadi, H., Howes, G. G., \& Dorland, W. 2014, Phys. Plasmas, 21, 020708, doi: http://dx.doi.org/10.1063/1.4867068

TenBarge, J. M., \& Howes, G. G. 2012, Phys. Plasmas, 19, 055901, doi: 10.1063/1.3693974

—. 2013, Astrophys. J. Lett., 771, L27, doi: 10.1088/2041-8205/771/2/L27
TenBarge, J. M., Howes, G. G., \& Dorland, W. 2013, Astrophys. J., 774, 139, doi: 10.1088/0004-637X/774/2/139

Vasyliunas, V. M. 1975, Reviews of Geophysics and Space Physics, 13, 303, doi: 10.1029/RG013i001p00303

Vech, D., Mallet, A., Klein, K. G., \& Kasper, J. C. 2018, The Astrophysical Journal Letters, 855, L27, doi: 10.3847/2041-8213/aab351

Vörös, Z., Yordanova, E., Varsani, A., Genestreti, K. J., \& Khotyaintsev et. al., Y. V. 2017, Journal of Geophysical Research: Space Physics, 122, 11,442, doi: 10.1002/2017JA024535

Wan, M., Matthaeus, W. H., Servidio, S., \& Oughton, S. 2013, Phys. Plasmas, 20, 042307, doi: 10.1063/1.4802985

Wan, M., Matthaeus, W. H., Karimabadi, H., et al. 2012, Phys. Rev. Lett., 109, 195001, doi: 10.1103/PhysRevLett.109.195001

Wilder, F. D., Ergun, R. E., Burch, J. L., Ahmadi, N., \& Eriksson et. al., S. 2018, Journal of Geophysical Research (Space Physics), 123, 6533, doi: 10.1029/2018JA025529

Wu, P., Perri, S., Osman, K., et al. 2013, Astrophys. J. Lett., 763, L30, doi: 10.1088/2041-8205/763/2/L30

Yordanova, E., Vörös, Z., Varsani, A., Graham, D. B., \& Norgren et. al., C. 2016, Geophys. Res. Lett., 43, 5969, doi: 10.1002/2016GL069191

Zenitani, S., Hesse, M., Kimas, A., \& Kuznetsova, M. 2011, Phys. Rev. Lett., 106, 195003

Zhdankin, V., Uzdensky, D. A., Perez, J. C., \& Boldyrev, S. 2013, Astrophys. J., 771, 124, doi: 10.1088/0004-637X/771/2/124 


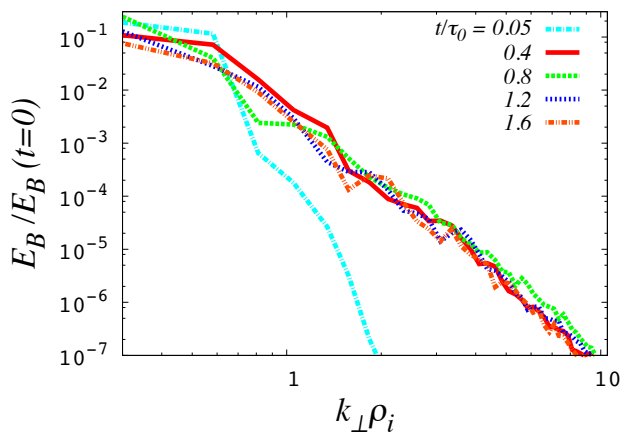

Figure A1. Evolution of the magnetic energy spectrum as a function of scale, showing a developed turbulent cascade in the dissipation range.

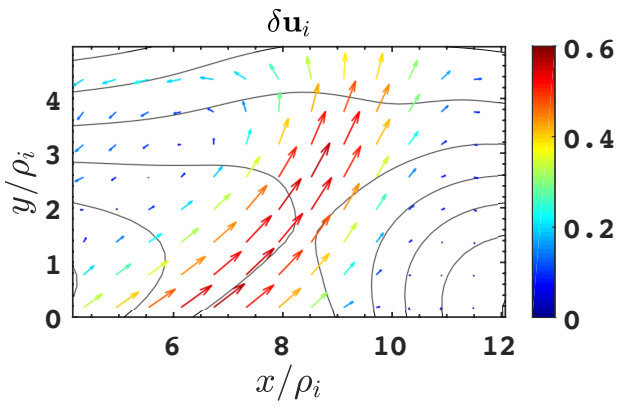

Figure A2. Ion flow velocity $\delta \mathbf{u}_{i}$ for X3, showing one outflow jet in ions. Compare with the electron flow velocity in Figure $4(\mathrm{c})$. $\delta \mathbf{u}_{i}$ is normalized to the ion thermal speed $v_{t i}$.

\section{APPENDIX}

Two supplementary figures are available. Figure A1 shows the turbulent cascade in the dissipation range in the magnetic energy spectrum. Figure A2 shows the fluctuating in-plane ion flow velocity $\delta \mathbf{u}_{i}$ for X3. 